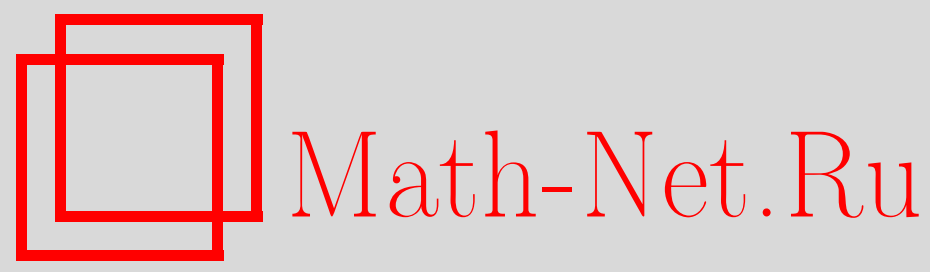

А. В. Рожков, Максимальные локально конечные подгруппы в группе Григорчука, Матем. заметки, 1998, том 63, выпуск 4, 617-624

DOI: https://doi.org/10.4213/mzm1322

Использование Общероссийского математического портала Math-Net.Ru подразумевает, что вы прочитали и согласны с пользовательским соглашением http://www . mathnet.ru/rus/agreement

Параметры загрузки:

IP : 54.237 .59 .107

26 апреля 2023 г., 09:32:55

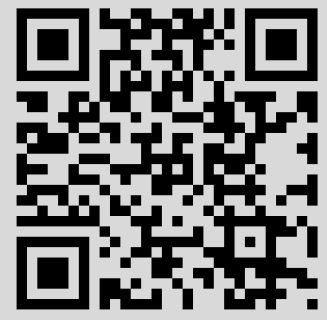




\section{МАКСИМАЛЬНЫЕ ЛОКАЛЬНО КОНЕЧНЫЕ ПОДГРУППЫ В ГРУППЕ ГРИГОРЧУКА}

\section{А. В. Рожков}

Явно строятся примеры максимальных локально конечных подгрупп в 3-порожденной 2-группе Григорчука, изучаются их свойства.

Библиограффия: 5 названий.

Речь идет о 3-порожденной 2-групе Григорчука $Г$ [1], активно изучаемой в последнее время. Р.И. Григорчук доказал, что она имеет промежуточньй рост, И. Г. Лысёнок описал ее определяюшие соотношения. К настоящему моменту описаны также ее конечные, абелевы, нормальные подгрупшы, найдены ряды коммутантов, нижний центральный ряд, описаны централизаторы и повторные централизаторы элементов [2] и т.д.

Поскольку эта группа нелокально конечна, представляет несомненньй интерес изучение ее максимальных локально конечных подгрупп. Их существование следует из абстрактной теории групп, однако построение конкретных примеров сопряжено с большими трудностями. Отметим также, что всякая максимальная локально конечная подгруппа в групе Григорчука, очевидно, совпадает со своим нормализатором, что дает еще один пример подобных подгрупп в группе $Г$. Ранее было известно, что самонормализуюшимися являются централизаторы инволюций [2] и стабилизаторы бесконечных путей [3].

Основным результатом статьи является явное построение максимальных локально конечных подгрупп $F, G, H$ и выяснение ряда их интересных свойств. Оказывается, что в них вкладьвается любая конечная 2-груша, что их централизаторы во всей группе автоморфизмов Aut $T$ дерева $T$ тривиальны и т.д.

В работе используются некоторые результаты статьи [2].

1. Основные соотношения. Группа Григорчука $\Gamma=\operatorname{gr}(c, f, g, h)$ задается четырьмя, фактически тремя, автоморфизмами бесконечного однородного 2-дерева $T$ :
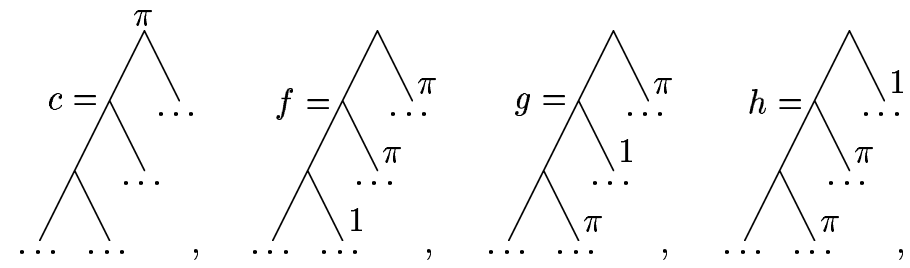

где $\pi=(0,1)-$ подстановка ребер, последовательности

$$
\pi, \pi, 1, \ldots, \pi, 1, \pi, \ldots, \quad 1, \pi, \pi, \ldots
$$


циклически повторяются, а во всех неотмеченных вершинах стоят тождественные подстановки. Из определения следует, что $c^{2}=f^{2}=g^{2}=h^{2}=1, f g=h, g h=f, h f=g$. Мы будем использовать следующие обозначения. Если $v-$ вершина, то $T_{(v)}$ - поддерево с начальной вершиной $v$. Деревья $T$ и $T_{(v)}$ естественньм образом изоморфны. Пусть $\mathrm{id}^{(v)}: T \rightarrow T_{(v)}$ - указанный изоморфизм, которьй мы будем обозначать через $\mathrm{id}^{(n)}$, если важна только длина $n$ вершины $v$. Указанньй изоморфизм естественньгм образом задает изоморфизм Aut $T \rightarrow \operatorname{Aut} T_{(v)}$ групп автоморфизмов деревьев, который мы будем обозначать теми же символами $\mathrm{id}^{(v)}$ и $\mathrm{id}^{(n)}$.

Если $X \leqslant$ Aut $T, V$ - некоторое подмножество вершин дерева $T$, то по определению

$$
\operatorname{st}_{X}(V)=\bigcap_{v \in V} \operatorname{st}_{X}(v), \quad \operatorname{cost}_{X}(V)=\operatorname{gr}\left(\bigcup_{v \in V} \operatorname{st}_{X}\left(T \backslash T_{(v)}\right)\right)
$$

- стабилизатор и костабилизатор множества $V$ соответственно. Нетрудно видеть, что $\operatorname{cost}_{X}(v) \triangleleft \operatorname{st}_{X}(v)$ и при $u \leqslant v$

$$
\operatorname{st}_{X}(v) \leqslant \operatorname{st}_{X}(u), \quad \operatorname{cost}_{X}(v) \leqslant \operatorname{cost}_{X}(u) .
$$

Кроме того, при несравнимых $u$ и $v$ костабилизаторы $\operatorname{cost}_{X}(u)$ и $\operatorname{cost}_{X}(v)$ поэлементно перестановочны.

Пусть $|v|$ - длина вершины $v$. Тогда по определению

$$
\operatorname{st}_{X}(n)=\bigcap_{|v|=n} \operatorname{st}_{X}(v), \quad \operatorname{cost}_{X}(n)=\operatorname{gr}\left(\bigcup_{|v|=n} \operatorname{cost}_{X}(v)\right) .
$$

Если $x \in \operatorname{st}_{X}(v)$, то сужение автоморфизма $x$ на поддерево $T_{(v)}$ будем обозначать $x_{(v)}$ и называть $v$-срезкой. Если $x \in \mathrm{st}_{X}(n)$, то автоморфизм $x$ однозначно определяется своими $v$-срезками, $|v|=n$, и естественно отождествление $x=\left(\ldots, x_{(v)}, \ldots\right) \circ \mathrm{id}^{(n)}$. Чаще всего мы будем пользоваться им при $n=1$, опуская в обозначении $\mathrm{id}^{(1)}$ верхний индекс.

В указанных обозначениях имеем $f=(g \circ \mathrm{id}, c$ oid $)$ или $f=(g, c)$ о id. Таким образом, $h=(f, 1) \circ \mathrm{id}, \operatorname{chc}=(1, f) \circ \mathrm{id}$.

Прямые вычисления показывают, что

$$
(c h)^{4}=(c g)^{8}=(c f)^{16}=1, \quad \operatorname{gr}(c, h) \cong D_{4}, \quad \operatorname{gr}(c, g) \cong D_{8}, \quad \operatorname{gr}(c, f) \cong D_{16} .
$$

Очевидно, $\operatorname{st}(1)=\operatorname{gr}(f, g, c f c, c g c)$, значит $\operatorname{st}(1)_{(v)} \cong \Gamma$ о id при $v=0,1$. Следовательно, $\operatorname{st}(v)_{(v)} \cong \Gamma$ о $\mathrm{id}^{(v)}$ для любой вершины $v$ дерева $T$.

Условимся, что в обозначениях стабилизаторов и костабилизаторов, если они рассматриваются во всей групше $Г$ или во всей групше $\Gamma$ о id $(n)$, мы будем опускать для краткости такие индексы.

Если $X-$ подмножество групшы $\Gamma$, то $\bar{X}^{\Gamma}$ - нормальное замькание множества $X$ в группе $\Gamma$. 
Лемма 1 [2]. Пусть $K={\overline{(c f)^{2}}}^{\Gamma}, L=\bar{f}^{\Gamma}$ - нормальные подгруппь. Тогда имеют место соотношения:

1) $K=\operatorname{gr}\left((c f)^{2},\left(1,(c f)^{2}\right) \circ \mathrm{id},\left((c f)^{2}, 1\right) \circ \mathrm{id}\right), L=\operatorname{gr}(f, K)$;

2) $\Gamma / L \cong D_{4} \cong \operatorname{gr}(c, h), \Gamma / K \cong D_{4} \times \mathbb{Z}_{2}, L / K \cong \operatorname{gr}(f) \cong \mathbb{Z}_{2}$;

3) $\operatorname{cost}(v)_{(v)}=L \circ$ id $n p u|v|=1$;

4) $\operatorname{cost}(v)_{(v)}=\operatorname{cost}_{K}(v)_{(v)}=K \circ \mathrm{id}^{(n)} n p u|v|=n>1$;

5) $(c g)^{4} \in K, K / \operatorname{cost}_{K}(1) \cong D_{4}$,

и всякий әлемент этой факторгруппь имеет вид $(\psi(c, g), \psi(g, c)) \operatorname{cost}_{K}(1)$, әде $\psi-$ некоторое слово от двух переменных.

Введем основной объект нашего изучения - подгрупшы $F, G, H$ :

$$
\begin{gathered}
F=\operatorname{gr}\left(c, f, a_{u}, b_{v}|| u|=3 n,| v \mid=3 n+1, n \in \mathbb{N}\right), \\
G=\operatorname{gr}\left(c, g, a_{u}, b_{v}|| u|=3 n+2,| v \mid=3 n, n \in \mathbb{N}\right), \\
H=\operatorname{gr}\left(c, h, a_{u}, b_{v}|| u|=3 n+1,| v \mid=3 n+2, n \in \mathbb{N}\right),
\end{gathered}
$$

где $a_{u}, b_{u} \in \operatorname{cost}(u)$ и $\left(a_{u}\right)_{(u)}=(c f)^{2} \circ \mathrm{id}^{(n)},\left(b_{u}\right)_{(u)}=(c g)^{4} \circ \mathrm{id}^{(n)}, n=|u|$. Ниже будет доказано, что подгрупшы $F, G, H$ максимальные локально конечные.

Отметим, что в силу утверждений 4), 1), 5) леммы 1 элементы $a_{u}, b_{v}$ действительно существуют в группе $Г$. Кроме того, при разных $u, v$ эти элементы различны, что влечет бесконечность подгрупп $F, G, H$.

\section{2. Основные леммы.}

ЛЕмма 2. Пусть и-вериина длины 1. Тогда имеют место равенства

$$
\operatorname{st}_{F}(1)_{(u)}=G \circ \mathrm{id}, \quad \operatorname{st}_{G}(1)_{(u)}=H \circ \mathrm{id}, \quad \operatorname{st}_{H}(1)_{(u)}=\operatorname{st}_{F \circ \mathrm{id}}(1)
$$

Доказательство очевидно.

Именно ввиду леммы 2 возникает необходимость рассматривать все три подгрупшы одновременно.

\section{ЛЕмма 3. Подгруппа $F$ локально конечна.}

ДокАЗАТЕЛЬСтво. Если вершины $u$ и $v$ несравнимы, то элементы $a_{u}$ и $a_{v}, b_{u}$ и $b_{v}$, $a_{u}$ и $b_{v}$ перестановочны. Если же $x$ - произвольный порождающий группы $F$, то имеют место равенства $a_{u}^{x}=a_{v}^{ \pm 1}, b_{u}^{x}=b_{w}$, где $u$ и $w$ - некоторые вершины длины $|u|$. Отсюда следует, что подгруппа $\operatorname{gr}\left(c, f, a_{u}, b_{v}|| u|| v \mid,<n\right)$ конечна для любого натурального числа $n$. Лемма доказана.

Аналогично доказывается локальная конечность подгрупп $G$ и $H$.

Лемма 4. Пусть $x=\left(x_{1}, x_{2}\right)$ - әлемент группь Г. Тогда имеют место соотношения:

1) если $x_{1}, x_{2} \in G \circ \mathrm{id}$, mo $x \in F$,

2) если $x_{1}, x_{2} \in H$ о $\mathrm{id}$, то $x \in G$,

3) если $x_{1}, x_{2} \in F \circ \mathrm{id}$, то $x \in H$. 
ДокаЗАтЕЛЬство. Докажем 1). Если $x_{1}, x_{2} \in \operatorname{gr}\left(a_{u}, b_{v}|| u|=3 n+2| v \mid,=3 n\right.$, $n \in \mathbb{N}$ ), то $x \in \operatorname{cost}_{F}(1)<F$. Поэтому с точностью до умножения на элементы из $\operatorname{cost}_{F}(1)$ можно считать, что $x_{1}, x_{2} \in \operatorname{gr}(c, g)$ о id.

Пусть $x_{i}=\psi_{i}(c, g)$ ○ id, где $\psi_{i}$ - некоторое слово от переменных $c$ и $g, i=1,2$. Тогда

$$
x \cdot \psi_{1}^{-1}\left(f^{c}, f\right)=\left(1, \psi_{2}(c, g) \cdot \psi_{1}^{-1}(c, g)\right) \circ \mathrm{id}=(1, \psi(c, g)) \circ \mathrm{id} .
$$

Следовательно, $\psi(c, g) \in L$ и в силу леммы $1 \psi(c, g) \in\left\{1,(c g)^{4}\right\}$. В любом случае $(1, \psi) \in \operatorname{cost}_{F}(1)$, и значит, $x \in F$.

Докажем 2). Как и выше, можно считать, что по модулю подгрупшы $\operatorname{cost}_{G}(1)$ имеет место включение $x_{1}, x_{2} \in \operatorname{gr}(c, h)$ оid, где $x_{i}=\psi_{i}(c, h)$ oid, $i=1,2$. Тогда $x \cdot \psi_{1}^{-1}\left(g^{c}, g\right)=$ $(1, \psi(c, h))$ o id. Так как $\operatorname{gr}(c, h) \cap L=1$, имеем $\psi(c, h)=1$. Значит, $x \in G$.

Перейдем к доказательству 3$)$. С точностью до умножения на элементы из cost $_{H}(1)$ можно считать, что $x_{1}, x_{2} \in \operatorname{gr}(c, f)$.

Так как $(f, 1)$ и $\left(f^{c}, 1\right)$ лежат в $\operatorname{cost}_{H}(1)$, по модулю подгрупшы $\operatorname{cost}_{H}(1)$ элемент $x$ равен $\left(c^{\varepsilon}, c^{\delta}\right)$ o id, где $\varepsilon, \delta \in\{0,1\}$. Поскольку единственным элементом такого вида в группе Г является $(1,1)$, лемма доказана.

Если $x$ - элемент группы $\Gamma$, то он может быть записан в виде $* c * \cdots * c *, * \in\{f, g, h\}$, где крайние звездочки могут отсутствовать. Длиной такой записи мы назовем число входящих в нее символов $c, f, g, h$. Длину самой короткой записи назовем длиной элемента $x$ и обозначим через $\partial(x)$.

Отметим для дальнейшего, что если $x \in \operatorname{st}(1)$, то число вхождений символа $c$ в любую запись элемента $x$ должно быть четным. Поэтому любая запись элемента из стабилизатора st(1) имеет один из следуюших типов:

$$
\underbrace{c * \cdots c *}_{4 k}, \underbrace{* c \cdots * c}_{4 k}, \underbrace{* c \cdots c *}_{4 k+1}, \underbrace{c * \cdots * c}_{4 k+3}, \quad k=0,1, \ldots
$$

Таким образом, длина $\partial(x)=n=4 k+2$ для элемента $x \in \operatorname{st}(1)$ невозможна.

Лемма 5. Пусть $x$ - әлемент подгруппы $K, d \in\{h, c h, h c, c h c\}$. Тогда подгруппа $X=\operatorname{gr}\left(\operatorname{st}_{F}(1), d x\right)$ нелокально конечна.

ДокАЗАТЕЛЬСТво проведем индукцией по $n=\partial(x)$. Так как $K \triangleleft \Gamma$ и подгруппа $\operatorname{st}_{F}(1)$ выдерживает сопряжения элементом $c$, то, в силу того что $h$ и $c h c, c h$ и $h c$ сопряжены элементом $c$, мы можем ограничиться только рассмотрением случаев $d=h$ и $d=c h$.

Пусть $n<7$. Так как $K<\operatorname{st}(1)$, реально возможны только значения $n=0,1,3,4,5$.

Если $n=0$, то $x=1$, и непосредственно проверяется (сужением на поддеревья), что подгруппа $X=\operatorname{gr}\left(\operatorname{st}_{F}(1), d\right)$ всегда нелокально конечна. Например, при $d=h$, сужая подгрупу $X$ на поддерево $T_{(0)}$, согласно лемме 1 получаем подгрупу $X_{(0)}=$ $\operatorname{gr}(G \circ \mathrm{id}, f \circ \mathrm{id})=\Gamma \circ \mathrm{id}$.

Если $n=1$ или $n=3$, то элемент $x$ не может принадлежать подгруппе $K$.

Если $n=4$, то с точностью до умножения на элементы из $\operatorname{st}_{F}(1)$ и взятия обратного $x=h c f c g=h(c f)^{2} h=(f c h, g c)$ oid. Непосредственно проверяется, что и в этом случае подгрупшы $\operatorname{gr}\left(\operatorname{st}_{F}(1), d x\right)$ нелокально конечны.

Пусть $n=\partial(x)>6$, и для слов длины меньшей $n$ утверждение доказано. 
Пусть $d=h, x=\left(x_{1}, x_{2}\right)$. Тогда в силу леммы 1

$$
X_{(0)}=\operatorname{gr}\left(G \circ \mathrm{id}, f \circ \mathrm{id} \cdot x_{1}\right), \quad \text { где } \quad x_{1} \equiv(c g)^{i}(\bmod K \circ \mathrm{id}), \quad i=0,1,2,3 .
$$

Очевидно, что

$$
\partial\left(x_{1}\right) \leqslant \begin{cases}{\left[\frac{n+1}{2}\right]} & \text { при } n \equiv 1(\bmod 4), \\ {\left[\frac{n}{2}\right]} & \text { при } n \equiv 0(\bmod 4), \\ {\left[\frac{n-1}{2}\right]} & \text { при } n \equiv 3(\bmod 4),\end{cases}
$$

где [· - целая часть числа. Домножая элемент $x_{1}$ на подходящий элемент $t \in G$ o id, получаем $y=x_{1} t \in K$ o id. При этом всегда можно добиться, чтобы выполнялось неравенство

$$
\partial(y) \leqslant \partial\left(x_{1}\right)+3 \leqslant\left[\frac{n+1}{2}\right]+3 .
$$

В самом деле, если $x_{1}$ имеет вид $\cdots * c$, то полагаем соответственно $t=g c,(c g)^{2}, c g$ при $i=1,2,3$. Аналогично, если $x_{1}$ имеет вид $\cdots c *$, то полагаем соответственно $t=g c$, $(g c)^{2}, c g$ при $i=1,2,3$.

Таким образом, $\partial(y) \leqslant[(n+1) / 2]+3$ при $n \equiv 1(\bmod 4)$ и $\partial(y) \leqslant[n / 2]+3$ в остальных случаях.

Теперь рассмотрим случай $d=c h$. Для проекции $X_{(0)}$ подгрупшы $X$ на поддерево $T_{(0)}$ имеет место включение

$$
X_{(0)}=\operatorname{gr}\left(G \circ \mathrm{id}, G^{x_{2}} \circ \mathrm{id}, f \circ \mathrm{id} \cdot x_{1} x_{2}\right) \geqslant \operatorname{gr}\left(G \circ \mathrm{id}, f \circ \mathrm{id} \cdot x_{1} x_{2}\right)
$$

Так как $x_{1} \equiv(c g)^{i}(\bmod K \circ \mathrm{id}), x_{2} \equiv(g c)^{i}(\bmod K \circ \mathrm{id}), i=0,1,2,3$, выполнено $x_{1} x_{2} \in K$ о id.

Оценим длину слова $y=x_{1} x_{2}$. Полагая $n=4 k+1$, имеем

$$
x=\left(x_{1}, x_{2}\right)=(\underbrace{* c \cdots c *}_{2 k+1}, \underbrace{c * \cdots * c}_{2 k+1}) .
$$

Если в произведении $y$ нет сокращений, то $\partial(y)=4 k+2$, что невозможно для элемента из подгрупшы $K$. Поэтому в записи хотя бы одного из элементов $x_{1}, x_{2}$ отсутствует хотя бы одна буква $c$. Но тогда соединяются две соседние звездочки и, тем самым, становится верным неравенство $\partial(y) \leqslant n-1$.

Если

$$
x=\underbrace{c * \cdots * c}_{4 k+3}=(\underbrace{c * \cdots * c}_{2 k+1}, \underbrace{* c \cdots * c}_{2 k+1}),
$$

то $\partial(y) \leqslant 4 k+2=n-1$.

Если

$$
x=\underbrace{c * \cdots c *}_{4 k}=(\underbrace{c * \cdots c *}_{2 k}, \underbrace{* c \cdots * c}_{2 k}),
$$

то $\partial(y) \leqslant 2 k+2 k-1=n-1$. 
Если

$$
x=\underbrace{* c \cdots * c}_{4 k}=(\underbrace{* c \cdots * c}_{2 k}, \underbrace{c * \cdots c *}_{2 k}),
$$

то $\partial(y) \leqslant 2 k+2 k-3=n-3$.

Таким образом, во всех случаях $\partial(y) \leqslant n-1$.

Объединяя полученные результаты, находим, что для доказательства леммы достаточно показать нелокальную конечность подгруппы $Y=\operatorname{gr}(G, f y)$, где $y \in K$ и

$$
\partial(y) \leqslant \begin{cases}\max \left\{n-1,\left[\frac{n+1}{2}\right]+3\right\} & \text { при } n \equiv 1(\bmod 4), \\ \max \left\{n-1,\left[\frac{n}{2}\right]+3\right\} & \text { при } n \equiv 0,3(\bmod 4) .\end{cases}
$$

Поскольку $n>6$, непосредственно проверяется, что в обоих случаях максимум равен $n-1$, т.е. всегда $\partial(y) \leqslant n-1$.

Пусть $y=\left(y_{1}, y_{2}\right)$. Тогда с точностью до изоморфизма id получаем

$$
Y_{(0)}=\operatorname{gr}\left(H, g y_{1}, c y_{2}\right)=\operatorname{gr}\left(H, f y_{1}, y_{2}\right) \text {. }
$$

Пусть $y=\left(y_{1}, y_{2}\right)=\left((c g)^{i},(g c)^{i}\right)\left(\bmod \operatorname{cost}_{K}(1)\right), i=0,1,2,3$.

Рассмотрим подгруппы, содержашиеся в группе $Y_{(0)}$ :

$$
\operatorname{gr}\left(H, f y_{1}\right)=\operatorname{gr}(H, f z) \quad \text { при } i=0,2, \quad \operatorname{gr}\left(H, y_{2}\right)=\operatorname{gr}(H, f z) \text { при } i=1,3,
$$

где $z=y_{1}, f y_{2} c h, y_{1}(c h)^{2}=y_{1}(h c)^{2}, f y_{2} h c$ при $i=0,1,2,3$ соответственно.

Нетрудно видеть, что $z \in K$ и $\partial(z) \leqslant \partial\left(y_{j}\right)+3, j=1,2$. Более того, в силу неравенств $(1)$ и $\partial(y) \leqslant n-1$ самое слабое неравенство $\partial\left(y_{j}\right) \leqslant[((n-1)+1) / 2]$ получаем только тогда, когда $n-1 \equiv 1(\bmod 4)$. Однако, в этом случае $n \equiv 2(\bmod 4)$, что невозможно. Таким образом, всегда $\partial(z) \leqslant[(n-1) / 2]+3$.

Для завершения доказательства осталось показать, что подгруппа $Z=\operatorname{gr}(H, f z)$ нелокально конечна для любого $z \in K$ такого, что $\partial(z) \leqslant[(n-1) / 2]+3$.

Пусть $z=\left(z_{1}, z_{2}\right)$. Тогда с точностью до изоморфизма id имеем $Z_{(0)}=\operatorname{gr}\left(\operatorname{st}_{F}(1), h z_{1}\right.$, $\left.c z_{2}\right)$. Пусть

$$
z=\left(z_{1}, z_{2}\right)=\left((c g)^{i},(g c)^{i}\right)\left(\bmod \operatorname{cost}_{K}(1)\right), \quad i=0,1,2,3 .
$$

Для каждого значения $i$ в группе $Z_{(0)}$ выберем свою подгруппу и докажем ее нелокальную конечность.

При $i=0$ это подгруппа $\operatorname{gr}\left(\operatorname{st}_{F}(1), h t\right)$, где $t=z_{1}$.

При $i=1$ это подгруппа $\operatorname{gr}\left(\operatorname{st}_{F}(1), c z_{2}\right)=\operatorname{gr}\left(\operatorname{st}_{F}(1), c h c \cdot t\right)$, где $t=c h z_{2} f$.

В оставшихся случаях рассмотрим подгруппы $\operatorname{gr}\left(\operatorname{st}_{F}(1), c z_{2}\right)=\operatorname{gr}\left(\operatorname{st}_{F}(1), h t\right)$, где $t=g c z_{2}$ при $i=2, t=h c z_{2} f$ при $i=3$.

Легко видеть, что во всех случаях $t \in K$ и вьполнены неравенства

$$
\partial(t) \leqslant \partial\left(z_{2}\right)+3 \leqslant\left[\frac{\partial(z)+1}{2}\right]+3 \leqslant\left[\frac{[(n-1) / 2]+4}{2}\right]+3 \leqslant\left[\frac{n}{4}\right]+5 .
$$

Все четыре рассмотренные нами подгрупшы являются подгруппами из формулировки леммы 5 , а именно, имеют вид $\operatorname{gr}\left(\operatorname{st}_{F}(1), d t\right)$, где $d \in\{h, c h c\}$ и $\partial(t) \leqslant[n / 4]+5$. Так как $n>6$, нетрудно видеть, что $[n / 4]+5<n$, и по предположению индукции каждая из этих подгрупп нелокально конечна. Лемма доказана. 


\section{3. Максимальные локально конечные подгруппы.}

Теорема 1. Подгруппа, строго содержащая любую из подгрупп $F, G, H$, нелокально конечна.

ДокАЗАТЕЛЬСТво. Пусть $X$ - одна из подгрупп $F, G, H, x \notin X$. Тогда индукцией по длине $\partial(x)$ слова $x$ докажем, что подгрупша $\operatorname{gr}(x, X)$ нелокально конечна. База индукции $\partial(x)=1$. В этом случае, очевидно, $\operatorname{gr}(x, X)=\Gamma$.

Пусть $x \notin F$. Так как $c \in F$, можно считать, что $x=\left(x_{1}, x_{2}\right)$. Согласно лемме 2 хотя бы один из элементов $x_{1}$ или $x_{2}$ не лежит в подгруппе $G$ o id. Поскольку $\partial\left(x_{i}\right)<\partial(x)$, $i=1,2$, по предположению индукции хотя бы одна из подгрупп $\operatorname{gr}\left(G\right.$ о $\left.\mathrm{id}, x_{i}\right), i=1,2$, нелокально конечна. Следовательно, нелокально конечна и исходная группа $\operatorname{gr}(x, F)$, так как подгрупшы $\operatorname{gr}\left(G\right.$ о id, $\left.x_{i}\right)$ являются ее факторами.

Если $x \notin G$, то нелокальная конечность подгруппы $\operatorname{gr}(x, G)$ доказывается аналогично.

Пусть $x=\left(x_{1}, x_{2}\right) \notin H$. Если $x_{i} \in \operatorname{st}(1) \backslash F$ о id для некоторого $i \in\{1,2\}$, то $x_{i}=\left(x_{i 1}, x_{i 2}\right)$, и по лемме $2 x_{i j} \notin G \circ \mathrm{id}^{(2)}$ для некоторого $j \in\{1,2\}$. Так как $\partial\left(x_{i j}\right)<\partial(x)$, по предположению индукции подгруппа $\operatorname{gr}\left(x_{i j}, G \circ\right.$ id $\left.^{(2)}\right)$ нелокально конечна.

Если же, например, $x_{1} \in \operatorname{st}_{F \text { oid }}(1)$, a $x_{2} \notin \mathrm{st}(1) \cup F$ o id, то $\left(x_{1}^{-1}, 1\right) \in H$, и значит, в группе Г существует элемент $\left(x_{1}^{-1}, 1\right) \cdot\left(x_{1}, x_{2}\right)=\left(1, x_{2}\right)$ такой, что $x_{2} \notin \operatorname{st}(1)$. Однако, в группе $Г$ таких элементов нет, поскольку cost $(1)<\mathrm{st}(1)$.

Последний из возможных случаев имеет место при $x_{1}, x_{2} \notin \operatorname{st}(1) \cup F$ o id. Согласно лемме 1 имеем $x=\left(x_{1}, x_{2}\right)=\left((c h)^{j} y,(h c)^{j} z\right)$, где $y, z \in L \circ \mathrm{id}, j=1,3$. Отметим, что $(c h)^{4}=1$, поэтому $(c h)^{3}=h c$. Так как $L / K \cong \operatorname{gr}(f)$ по лемме 1 , домножая, если необходимо, элемент $x$ на элементы из групшы $H$, можно считать, что $x_{1}=c h y$ или $x_{1}=h c y$, где $y \in K$ о id. Теперь с учетом леммы 5 заключаем, что подгруппа $\operatorname{gr}\left(x_{1}, \operatorname{st}_{F \text { oid }}(1)\right)$ нелокально конечна, значит такова и подгруппа $\operatorname{gr}(x, H)$. Теорема доказана.

\section{Теорема 2. Имеют место соотношения:}

1) $F \cap G=G \cap H=F \cap H=\operatorname{gr}(c)$;

2) $\bar{F}^{\Gamma}=\operatorname{gr}(c, f, g c g), \bar{G}^{\Gamma}=\operatorname{gr}(c, g, h c h), \bar{H}=\operatorname{gr}(c, h, f c f)-$ nодәруппь индекса 2 ;

3) чентрализаторы подгрупп $F, G, H$ в группе Aut $T$ автоморфизмов дерева $T$ тривиальны.

ДокАЗАтЕльство. Пусть $x \in F \cap G$. Доказательство проведем индукцией по $\partial(x)$. Если $\partial(x)=1$, то утверждение 1 ) очевидно.

Если $x \notin \operatorname{st}(1)$, то вместо элемента $x$ возьмем элемент $y=x c \in F \cap G \cap \operatorname{st}(1)$. Поэтому пусть $y=\left(y_{1}, y_{2}\right)$. Тогда в силу леммы $2 y_{1}, y_{2} \in(G \cap H) \circ \mathrm{id}$. Taк как $\partial\left(y_{i}\right)<\partial(x)$, $i=1,2$, по предположению индукции $y_{1}, y_{2} \in \operatorname{gr}(c \circ \mathrm{id})$. Поскольку в группе Г нет элементов вида $(1, c)$ o id, $(c, 1) \circ \mathrm{id},(c, c)$ о id, имеем $y_{1}=y_{2}=1$. Следовательно, $x=1$ или $x=c$.

Аналогично доказываются утверждения для остальных пересечений из 1).

Докажем утверждение 2). Очевидно, что все интересующие нас нормальные замыкания содержат подгруппу $\bar{c}^{\Gamma} \geqslant \overline{\{[c, f],[c, g],[c, h]\}}^{\Gamma}$, а значит, и коммутант групш $\Gamma$. Столь же очевидно, что, факторизуя рассматриваемые нормальные замькания по коммутанту, мы получаем групшы порядка 4. Так как индекс коммутанта равен 8 [2], все 
рассматриваемые нами нормальные замыкания имеют в группе Г индекс 2 . Теперь из списка семи подгрупп индекса 2, приведенного в [2], достаточно выбрать необходимые.

Пусть $X$ - одна из подгрупп $F, G, H$. Докажем чуть более сильное утверждение, чем сформулированное в 3). А именно:

4) централизатор подгрупш $\mathrm{st}_{X}(1)$ тривиален в $\mathrm{Aut} T$.

Пусть элемент $x=c\left(x_{1}, x_{2}\right)$ централизует подгруппу $\mathrm{st}_{X}(1)$. Тогда получаем противоречие

$$
\left(x_{1}^{-1}, x_{2}^{-1}\right) c \cdot(g, c) \circ \mathrm{id} \cdot c\left(x_{1}, x_{2}\right)=\left(c^{x_{1}}, c^{x_{2}}\right) \circ \mathrm{id} \neq(g, c) \circ \mathrm{id},
$$

так как порождающие $c$ и $g$ несопряжены. Следовательно, централизатор подгруппы st $F(1)$ лежит в подгрупше st(1). Аналогично доказывается, что централизаторы подгрупп $\operatorname{st}_{G}(1)$ и $\mathrm{st}_{H}(1)$ лежат в $\mathrm{st}(1)$.

Однако, если $x=\left(x_{1}, x_{2}\right)$ централизует, например, подгруппу $\operatorname{st}_{F}(1)$, то $x_{1}$ и $x_{2}$ централизуют подгруппу $G$ oid, и значит, $x_{1}, x_{2} \in \operatorname{st}_{\Gamma \text { oid }}(1)$. Следовательно, $x \in \operatorname{st}(2)$ и т.д. Таким образом, $x=1$.

Так как для любой вершины $u$ костабилизатор $\operatorname{cost}_{X}(u) \neq 1$, используя метод доказательства теоремы об описании конечных подгрупп $A T_{\omega}$-групш, изложенньй в [4], можно доказать утверждение 4). Теорема доказана.

Отметим, что в произвольной финитно аппроксимируемой 2-группе централизатор любой конечной подгруппы бесконечен. Поэтому утверждение 3 из анонса [5] ошибочно.

В первом варианте статьи доказьвалась теорема о том, что централизатор любой конечной подгрупшы 2-групшы Григорчука содержит подгруппу, изоморфную некоторому костабилизатору группы Григорчука (в частности, подгруппу, изоморфную всей группе Григорчука). Это более сильное утверждение, чем просто бесконечность централизатора. Однако, аналогичная теорема справедлива для многих $A T$-групп, а не только для централизаторов конечных подгрупп 2-группы Григорчука. Мы докажем эту теорему в одной из следующих статей.

Автор благодарит рецензента за замечания, позволившие сократить объем статьи и избежать дублирования известных фактов.

\section{СПИСОК ЦИТИРОВАННОЙ ЛИТЕРАТУРЫ}

[1] Григорчук Р.И. К проблеме Бернсайда о периодических группах // Функцион. анализ и его прилож. 1980. Т. 14. № 1. С. 53-54.

[2] Рожков А. В. Централизаторы элементов в одной группе автоморфизмов деревьев // Изв. РАН. Сер. матем. 1993. Т. 57. №6. С. 82-105.

[3] Рожков А. В. О стабилизаторах кортежей в группах алешинского типа // Матем. заметки. 1990. T. 47. № 3. C. 121-128.

[4] Рожков А. В. О подгруппах некоторых групп алешинского типа // Алгебра и логика. 1986. T. 25. №6. C. $643-671$.

[5] Рожков А. В. Централизаторы и самонормализующиеся подгруппы в группе Григорчука // Международная конференция по алгебре. Тезисы докл. по теории групп. Новосибирск, 1991. C. 86 . 